\title{
Deporte y neoliberalismo en América Latina: los casos de Argentina y Brasil
}

\author{
Sports and neoliberalism in Latin America: the cases of Argentina and Brazil \\ Esportes e neoliberalismo na América Latina: os casos da Argentina e do Brasil \\ Eloy Altuve-MeJía ${ }^{1}$ \\ UNIVERSIDAD DEL ZULIA, LUZ, MARACAIBo, VENEZUELA
}

\begin{abstract}
RESUMEN
La elección de Macri, en Argentina, 2015, y el impeachment de Rouseff, en Brasil, 2016, significaron el retorno del neoliberalismo con fuerza en América Latina. En ambos casos, el deporte y particularmente la gestión de deportes tuvieron un papel importante: En Argentina, el desempeño exitoso de Macri como presidente-gerente de Boca Juniors fue el trampolín para su entrada en la política, marcó su carrera e incorporó a la campaña elecciones. En Brasil, la gestión de la Copa del Mundo de 2014 y de los Juegos Olímpicos 2016 concentró beneficios en la FIFA, en el COI, en socios transnacionales y emprendedores nacionales asociados a funcionarios públicos corruptos y políticos, haciendo del deporte un elemento movilizador que contribuyó a las protestas de 2013 y cuestionamiento del carácter popular-democrático del gobierno de Rouseff, al evidenciar el mantenimiento de beneficios a los poderosos, contribuyendo con su debilitamiento político.
\end{abstract}

Palabras clave: Deporte. Neoliberalismo. América Latina.

\begin{abstract}
The election of Macri, in Argentina, 2015, and the impeachment of Rouseff, in Brazil, 2016, marked the return of neoliberalism with strength in Latin America. It goes to show that in both cases sports and particularly sports management, played an important role: In Argentina, the successful exercise of Macri as president-manager of Boca Juniors was the springboard for his entry into politics, has marked his career and incorporated it to the electoral campaign. In Brazil, the management of the 2014 World Cup and Olympic Games 2016 concentrated its benefits on FIFA, the IOC, its transnational partners and national entrepreneurs associated with corrupt state officials and politicians, making sports a mobilizing element that contributed to the protests of 2013 and questioned the popular-democratic character of the government of Rouseff, evidencing that it maintained the benefits of the powerful, while deceiving its people because it did not fulfill what was promised and aggravated its problems, contributing to its political weakening.
\end{abstract}

Keywords: Sport. Neoliberalism. Latin America.

\section{RESUMO}

A eleição de Macri, na Argentina, 2015, e o impeachment de Rouseff, no Brasil, 2016, significaram o retorno do neoliberalismo com força na América Latina. Em ambos os casos, o esporte e particularmente o gerenciamento de esportes tiveram um papel importante: Na Argentina, o desempenho bem sucedido de Macri como presidentegerente do Boca Juniors foi o trampolim para sua entrada na política, ele marcou sua carreira e incorporou-o à campanha eleitoral. No Brasil, a gestão da Copa do Mundo de 2014 e dos Jogos Olímpicos 2016 concentrou benefícios na FIFA, no COI, em parceiros transnacionais e empreendedores nacionais associados a funcionários públicos corruptos e políticos, tornando o esporte um elemento mobilizador que contribuiu para os protestos de 2013 e questionamento do caráter popular-democrático do governo de Rouseff, ao evidenciar manutenção de benefícios aos poderosos, contribuindo com seu enfraquecimento político.

Palavras-chave: Esporte. Neoliberalismo. América Latina.

\footnotetext{
${ }^{1}$ Profesor de la Universidad del Zulia. E-mail: eloyaltuve@ hotmail.com
} 


\section{INTRODUCCIÓN}

El propósito de este trabajo es demostrar que el deporte y particularmente la gestión deportiva, desempeñó un papel importante en el retorno del neoliberalismo en Brasil y Argentina, en el siglo XXI.

Se inicia con la definición del deporte como Empresa Transnacional Atípica de Espectáculo y Entretenimiento (EDTEE), identificando sus componentes y explicando las relaciones que establecen entre sí. Determinándose, además, la distribución y concentración del poder en su interior.

Inmediatamente se explica como el Mundial 2014 y los Juegos Olímpicos (JO) 2016 en Brasil, concentraron sus beneficios en los organizadores-gestores (FIFA y el Comité Olímpico Internacional o COI) y sus Socios transnacionales, y en empresarios nacionales asociados con funcionarios estatales y políticos corruptos. Al mismo tiempo que las promesas de beneficios, progreso, ventajas económicas etc., que hicieron el gobierno, la FIFA y el COI al país no se cumplieron, creándose una gran molestia y enojo en amplios sectores de la población.

Se analiza la incorporación del amplio descontento producido por el Mundial 2014 (expresado a través de denuncias de los elevados costos que tenía para las arcas públicas el evento y cuestionamientos a su realización, quejas contra la FIFA etc.), como elemento movilizador que coadyuvaron a las masivas y multitudinarias protestas en 2013, articulándose con exigencias de solución de necesidades básicas, demandas de reivindicaciones muy específicas para mejorar la calidad de vida. Para demostrar que ese rechazo al macroevento contribuyó con el cuestionamiento (o puesta en duda) del carácter popular-democrático del gobierno de Dilma Rouseff, cuya aprobación cayó a la mitad y luego su reelección en 2014 fue muy estrecha; contribuyó con su debilitamiento político e incidió favorablemente en su destitución en 2016, episodio que es el punto de quiebre de la democracia brasileña y marca el retorno del neoliberalismo a Brasil con Michel Temer en el poder.

Luego, en la continuidad y profundización del proyecto neoliberal en Brasil con la victoria de Jair Bolsonaro, en 2018, se determina el lugar prominente ocupado por el deporte en su campaña electoral, expresado -en los medios convencionales y especialmente los digitales- como respaldo a la candidatura de famosos deportistascompetidores, activos y retirados de diferentes disciplinas deportivas y como registro de la presencia del candidato en partidos de fútbol en distintas ciudades y estadios, alentando a uno de los protagonistas.

En Argentina, se pretende explicar el papel del deporte en la victoria presidencial de Mauricio Macri, en 2015, que significa el retorno al neoliberalismo. Se registra el ejercicio de Macri como presidente-gerente exitoso del Club de Fútbol Boca Juniors (el más emblemático y popular del país) del 03-12-1995 al 2007, posición que impulsó su ingreso al terreno político y ha signado su carrera política, acompañándole siempre de manera tácita y/o expresa. Particularmente, se determina la incorporación destacada de dos elementos de la gerencia deportiva y/o vinculados con ella a la triunfante campaña electoral 2015:

- Extrapolación desde el deporte del concepto de gestión para ser convertido en el centro de la propuesta político-electoral.

- Combinación magistral de la imagen de Macri como político igual a cualquier ciudadano, con la dotación de una épica y heroísmo único -apuntalados por su labor gerencial en el Boca Juniors- que lo diferenciaba de los demás candidatos y lo convertía en el acreedor de las condiciones para ser presidente de la nación. 
Finalmente, consideramos que para tener una mejor comprensión de lo político en nuestros países, es necesario incorporar el elemento deportivo a su estudio, es decir, profundizar en el análisis de la relación deporte-poder, deporte-política etc.

\section{GESTIÓN Y FISONOMÍA DEL DEPORTE CONVERTIDO EN EMPRESA TRANSNACIONAL ATÍPICA DE ESPECTÁCULO Y ENTRETENIMIENTO (EDTEE)}

Junto al petróleo, comunicaciones e industria automovilística, el deporte actualmente es la cuarta actividad económica mundial lícita.

En EEUU, “en 2006 movilizó 213 mil millones US\$ y generó 2 y 7 veces más ganancias que las industrias automotriz y cine", respectivamente. En Europa, en 2015 generó " $1,76 \%$ del valor añadido bruto y $2,12 \%$ del empleo", estimándose que tiene un efecto multiplicador para la economía de 1,22 (ALTUVE-MEJÍA, 2019, p.132).

Mundialmente en 2014 generó "1\% del PIB". Los ingresos de la venta de entradas y acuerdos de emisión y patrocinio se aproximaron a "80.000 millones US\$..., que con la venta de mercancías, artículos, ropa y equipamiento alcanzaron 700.000 millones US\$"; su valor fue estimado en "1,5 billones US\$" (ALTUVE-MEJÍA, 2019, p. 132133). En 2015:

- El patrocinio fue de 57.500 millones US\$: Nike ocupó un relevante papel, “con ingresos de 30.600" es la marca que más factura. En los Clubes de EEUU se destacan: NHL (Adidas se convirtió en patrocinante por "490 en 7 años"); NFL (renovó con AB InBev por "1.400 durante 6 años"; NBA (Nike por "1.000 será su proveedor desde 2017", AB InBev firmó un contrato por 4 años y Verizon pagará "400 por 3 años") (ALTUVE-MEJÍA, 2019, p. 133). Otros acuerdos son los de los JO 2020, Asociación de Tenis Profesional-Emirates, Red Bull-TAG Heuer (F1), Toyota-Comité Paralímpico Internacional (ALTUVE-MEJÍA, 2019, p. 133).

- Derechos de TV: En la Liga de Fútbol Española (LIGA, 2019) por 3 años: Movistar pagará "825 millones US\$ por el mejor encuentro de la jornada" y Mediapro "2.090 por el principal paquete de partidos"; Orange y Vodafone por "330 más 33 en variables", transmitirán en los bares; internacionalmente en 2015 han ascendido a “690,8 millones US\$ que en los próximos ejercicios comercializará Mediapro”. En la Premier League de Inglaterra (PREMIER, 2019), "para 2016-2019, Sky y BT ofrecieron 7.700 millones US\$", $70 \%$ más que su anterior contrato.

El deporte es una empresa cuyo objetivo es producir un espectáculo cotidiano, masivo, y rentable. Funciona con la lógica, dinámica y orientación de cualquier transnacional, con las siguientes especificidades: "su área de influencia es el mundo; es completamente monopólica porque no existen dos o más versiones del mismo evento al mismo tiempo; sus componentes funcionan de manera independiente $\mathrm{e}$ interdependiente" (ALTUVE-MEJÍA, 2016, p. 69).

La función ideológica con la que nace el deporte moderno a fines del siglo XIX, se amplió con la intervención de los medios de comunicación, produciendoreproduciendo las siguientes ideas legitimadas institucionalmente y socialmente aceptadas: Rendimiento-productividad-rentabilidad-progreso lineal e infinito, EstadoNación; Perfección física sustentada en la ciencia y la tecnología, registrada en el récord y materializada en el campeón; Perfección colectiva o bienestar para todo(a)s alcanzable al contemplar el espectáculo y consumir objetos-mercancías inherentes y/o vinculados al deporte (ALTUVE-MEJÍA, 2016).

Concebir el deporte como espacio social universal de igualitarismo absoluto, modelo ideal de convivencia humana, resume la ideología deportiva globalizadora. Se 
presenta como un archipiélago de felicidad en medio de una tormenta de infelicidad que es el resto de la sociedad, convirtiéndose en el faro iluminador al cual deben seguir las demás instituciones sociales; pasando a ser fuente de consuelo, esperanza y resignación, porque -a pesar de las abismales diferencias sociales- en el deporte todos somos iguales y el tránsito a la felicidad no está tan lejos, se logrará en la medida que el resto de la sociedad se parezca cada vez más al deporte (ALTUVE-MEJÍA, 2018).

El deporte es un aparato ideológico estatal porque el Estado se legítima en un proceso único, indivisible y simultáneo de definición, formulación y ejecución de la política pública y de producción, reproducción y difusión de la ideología deportiva globalizadora (ALTUVE-MEJÍA, 2018).

\section{PAPEL DEL DEPORTE EN EL RETORNO DEL NEOLIBERALISMO EN BRASIL: DESTITUCIÓN DE DILMA ROUSEFF Y RETORNO DEL NEOLIBERALISMO}

Como parte de su política pública deportiva, Lula da Silva logró la asignación para Brasil de las sedes del Mundial y JO. La población apoyó ambos eventos porque el gobierno, la FIFA y el COI prometieron que producirían amplios beneficios, progreso, orgullo y ratificaban la condición de potencia del país. Pero, lo prometido no se cumplió y el apoyo-credibilidad popular fue desmoronándose.

Se va inmediatamente a demostrar como la organización-gestión del Mundial y JO y otros fenómenos asociados a ambos eventos, contribuyeron con el progresivo retiro del apoyo popular que tenían.

Desde la preparación del Mundial y JO se evidenció el poco poder de Brasil en su organización-gestión, su subordinación a la FIFA y el COI y sus Socios transnacionales patrocinantes. Ni el Estado ni los empresarios y mucho menos el resto de la sociedad, tuvieron poder en las decisiones fundamentales sobre ambos eventos.

El Estado cedió su jurisdicción y ámbitos de competencia con la aprobación por el Congreso -en octubre 2011- de la Ley 12.663 o Ley General de la Copa. Otorgó privilegios fiscales, comerciales y amplias y diversas competencias a los organizadoresgestores de ambos eventos y sus patrocinantes transnacionales (ALTUVE-MEJÍA, 2018).

En el Mundial Brasil dejó de percibir -al menos- 680 millones US\$ al exonerar del pago de impuestos sobre sus beneficios durante 4 años a la FIFA y sus Socios transnacionales. Romario de Souza, antigua estrella del fútbol metido en política, declaró que la FIFA “ingresaría 1.800 millones, lo que normalmente generaría unos 450 millones en impuestos fiscales, el servicio tributario interno de Brasil estimó la pérdida en 248,7 millones" (ZIMBALIST, 2016, p. 125). Otras exenciones para la FIFA fueron "relativas a la importación de materiales y equipos de televisión, además de otros impuestos sobre la venta". Durante el evento "la Agencia Tributaria no ingresó nada por los premios concedidos" (ZIMBALIST, 2016, p. 208-209).

Las exigencias -alegando razones técnicas - de la FIFA incluyeron al estadio Maracaná de Río de Janeiro, que fue demolido y reconstruido "para expandir su zona VIP y albergar 74.000 personas, lo que costó 500 millones US\$”. A pesar de que había sido "remodelado por 200 millones US\$ para los Juegos Panamericanos 2007" (ALTUVE-MEJÍA, 2018, p. 464). Y se extendieron hasta la construcción del nuevo estadio en Sao Paulo -rechazando el venerable estadio Morumbi - cuyo "costo total estimado...superaría con creces los 650 millones US\$” (ZIMBALIST, 2016, p. 120121). 
En los JO el COI y sus Socios transnacionales fueron exonerados del pago de impuestos por 4 años, abarcando también la exoneración a "contribuciones sociales y aranceles de importación en actividades propias y directamente vinculadas a la organización", que regirá hasta el 31 de diciembre de 2017. Se estima que el Estado dejó de "percibir 530 millones US\$ y algunas estimaciones lo ubican en 1.000 millones US\$” (ALTUVE-MEJÍA, 2018, p.465).

El costo del Mundial y JO duplicó lo estimado inicialmente, el financiamiento fue principalmente público y la prometida inversión privada no se produjo.

El Estado estima la inversión en "21 mil millones US\$" y fuentes independientes serias la estiman en "35 a 40 mil millones US\$” (ZIMBALIST, 2016, p. 208-209).

El costo final de los eventos todavía no ha sido precisado, pero en todo caso es mucho más alto de las apreciaciones estatales porque falta incluir: a) El sobreprecio en la construcción y remodelación de estadios e infraestructura, resultado de la corrupción de políticos y empresarios, favorecida por la aprobación en agosto 2011 de la no divulgación del presupuesto estatal antes de la licitación. Las contratistas del Mundial y JO "se encontraban bajo investigación judicial y con algunos presidentes detenidos en junio 2015" (ALTUVE-MEJÍA, 2018, p. 476). b) La inversión para competir y ganar la sede de los eventos. Luego viene el plan, asesorías, folletos videos promocionales, viajes a las reuniones del COI y la FIFA, recibimiento de sus ejecutivos etc. El Comité Organizador de los JO de Río contrató a AECOM de Los Ángeles, Wilkinson Eyre de Reino Unido y Pujol Arquitectura de Barcelona, IMG y McKinsey de Nueva York, para diseñar una estrategia y estilo urbanos para los juegos. c) La inversión en las ceremonias de apertura y clausura. Suelen ser astronómicas, China invirtió 343 millones US\$ (precios 2014) en la apertura de los JO 2008 (ALTUVE-MEJÍA, 2018).

La esperanza e ilusión de progreso y ventajas, destacadas por la FIFA, el COI y el gobierno con el argumento del impacto positivo para el desarrollo económico por la inversión privada, progresivamente se fue desvaneciendo.

"El capital privado centró su limitada inversión en sectores como el aeroportuario, donde invirtió 1.320.000.000 US\$" (ALTUVE-MEJÍA, 2018, p. 471).

El efecto del Mundial las autoridades lo estimaron en 2010, "en 58.080 millones US\$ y la creación de 3.500 .000 empleos", y en 2014, se estimó una inyección a la economía de "180 mil millones US\$ en los próximos 3 años producto del efecto rebote de la inversión”. Pero, más de "300.000 Vendedores Ambulante fueron afectados desde antes del Mundial al ser desalojados de lugares requeridos para construcción de infraestructuras"; en las ciudades donde se disputarían partidos no se expidieron nuevas licencias para la venta ambulante y las existentes fueron retiradas o no renovadas. "Otras fuentes consideraron que el empleo no superaría 300.000 plazas" (ALTUVEMEJÍA, 2018, p.468-469).

En los JO sería negativo porque apenas haría crecer " $0,05 \%$ al PIB, del que se esperaba una disminución de 3,5\% en 2016". La inversión en infraestructura y el gasto público adicional "incrementarán la deuda pública en 0,04\% del PIB". Se creará empleo en el corto plazo, por aumento de nuevas compañías, especialmente pequeñas; generación de presiones inflacionarias hasta 2020, "1\% de la inflación general en 2016 (estimada en $8,6 \%$ ), de la cual $0,4 \%$ es residual del Mundial, $0,4 \%$ de los JO y $0,2 \%$ es resultado de ser sede de ambos eventos en un tiempo muy corto" (ALTUVE-MEJÍA, 2019, p.163).

Para disponer de los espacios necesarios para las obras del Mundial y JO, se adelantaron reconversiones urbanísticas signadas por el desplazamiento forzoso y violento de pobladores de las favelas o barrios pobres, creándose una gran especulación inmobiliaria y agravando el problema de la vivienda. 
Para el Mundial se estiman en "250.000 los desalojos; en los JO, por su ubicación junto a las 4 áreas olímpicas, varias favelas fueron desalojadas para hacer construcciones más caras" (ALTUVE-MEJÍA, 2017, p. 60).

El precio de los inmuebles aumentó desproporcionadamente. De 2005 a 2014, el crédito inmobiliario y construcción civil aumentó 20 veces; en Sao Paulo y Río de Janeiro el precio del metro cuadrado se cuadriplicó. "Y en ese mismo periodo, había cerca de 6 millones de pisos desocupados, con 7 millones de familias sin techo", y el gobierno en vez de aumentar la oferta de casas populares, "financió a las de la construcción privada -con un espléndido beneficio- de urbanizaciones en áreas periféricas, cobrando alquileres más caros de lo que los más pobres pueden pagar", y simultáneamente apoyó "a las autoridades locales en los desalojos de ocupaciones" (GENTILI, 2016, p. 40-41).

Las promesas incumplidas echaron por tierra los argumentos favorables para el Mundial y los JO, traduciéndose en creciente pérdida de credibilidad del gobierno. Con el orgullo y soberanía nacional vapuleados por la FIFA y sus socios transnacionales, con el progreso económico prometido convertido en espejismo debelado por el alto costo del Mundial financiado con fondos públicos, inutilidad social de algunas obras y corrupción combinada con impunidad en buena parte de todas, y con una violenta política estatal de desalojos y reconversiones urbanísticas que profundizó el problema de la vivienda, se fue desmoronando la ideología deportiva mundialista en medio de una situación económica cada vez más difícil que agravaba los problemas de la vida cotidiana. La mayor parte de la población desmejorada económicamente entendió y sintió que el Mundial no le ayudaba en su vida, sintiéndose estafada y engañada. El gobierno invirtió sus ingresos cada vez más escasos (por la caída de los precios de los productos de exportación) en el Mundial, beneficiando principalmente a la FIFA, sus Socios transnacionales y empresarios constructores corruptos asociados con políticos y funcionarios estatales sobornados (ALTUVE-MEJÍA, 2019).

Las masivas protestas de 2013 expresaron el descontento popular, comprendían quejas contra los elevados costos del Mundial y contenían demandas de mejoría de la calidad de vida de la población, se articularon exigencias de solución de necesidades básicas y severos cuestionamientos a la realización del evento, a sus símbolos y a la FIFA: la aprobación del gobierno cayó a la mitad; la consigna Padrón FIFA mostraba lo que un sector extendido de la juventud y el pueblo trabajador "reclamaba para el transporte público, educación, salud, estándares similares al tratamiento que se le daba al uso de recursos públicos a las obras y organización" del Mundial y los JO. "Se extendieron rápidamente hasta que el 20 de junio casi 400 ciudades, incluyendo 22 capitales, salieron en manifestaciones y marchas que agruparon a más de un millón de personas" (CARCIONE, 2018).

La intensificación de la violencia y represión de las protestas 2013 aunado al intensivo y extensivo "despliegue de seguridad -el mayor de todos los Mundiales - del Mundial 2014", generó más rechazo por parte de importantes sectores de la población, "que expresó su descontento en las elecciones presidenciales de 2014 donde Dilma Rouseff es reelecta con una estrecha ventaja del 3\%" (ALTUVE-MEJÍA, 2019, p. 167).

Colapsada la ideología deportiva, intensificada la crisis económica y a flor de piel el descontento y enojo de sectores importantes de la población, la gran burguesía con el apoyo del capital transnacional inicia su demoledora ofensiva final desde mediados del 2015 e impide a Dilma Rouseff concluir su segundo gobierno cuyo ejercicio inició muy débil políticamente, continuó con los preparativos de los JO 2016 y el 12-05-16 es suspendida ( 2 años después del Mundial 2014), sustituida por un Presidente provisional 
que los inaugura el 05-08-16, los juegos concluyen el 21-08-16 y es destituida el 31-0816 (ALTUVE-MEJÍA, 2019).

Fue prominente lugar del deporte en la victoriosa campaña electoral de Bolsonaro en 2018 y la profundización del proyecto neoliberal. Él logró articular simultáneamente el respaldo a su candidatura de famosos deportistas-competidores (activos y retirados) de fútbol, automovilismo, volibol, lucha, con su presencia en partidos de distintas ciudades y estadios, alentando a uno de los protagonistas, posó con la camiseta - sin distinción de categorías- de al menos una docena de Clubes (ALTUVE-MEJÍA, 2019).

Convirtió al deporte en uno de los escenarios privilegiados donde mejor operó la auténtica revolución tecno-política de su campaña electoral. Se registró, difundió y propagó masivamente a través de las redes sociales su presencia y protagonismo en los estadios y el apoyo de deportistas-competidores, en fin, el respaldo del mundo deportivo (ALTUVE-MEJÍA, 2019).

\section{PAPEL DEL DEPORTE EN EL RETORNO DEL NEOLIBERALISMO EN ARGENTINA}

Para Mauricio Macri ejercer del 1995 al 2007 la presidencia del Club de Fútbol Boca Juniors, el más emblemático y popular del país, fue el trampolín para incursionar como político. Su gestión fue calificada unánimemente como exitosa porque ganó 17 títulos (4 Copas Libertadores de América y 2 Intercontinentales) y la Federación Internacional de Historia y Estadística de Fútbol reconoció al Club en 2010 como el mejor de la década (ALTUVE-MEJÍA, 2018).

De su gestión deportiva, entre otros, aprendió-cultivó tres elementos que incorporó a su carrera política: 1) Entendió y asumió el deporte como apolítico, neutral. El Boca Juniors fue el ensayo exitoso de un proyecto "no político", "no ideológico", fundamentado en la "gestión". 2) Descubrió que el campeón es en el deporte lo que el candidato es en la política: modelo típico-ideal, sintetiza esfuerzo, sacrificio y trabajo en equipo, es percibido próximo a la gente y simboliza transparencia total al ganar porque es el mejor. 3) Utilización de los medios de comunicación. (ALTUVE-MEJÍA, 2018).

Estos aprendizajes, junto a otros provenientes del ámbito político y demás escenarios sociales, le permiten a Macri obtener su primera gran victoria como Jefe de Gobierno de la Ciudad de Buenos Aires entre 2007-2011, incorporándolos con amplitud a la campaña electoral presidencial 2015 , como se va a demostrar inmediatamente.

El centro de su propuesta política fue la gestión, desprovista de ideología, ascética y neutral, promotora del apoliticismo y encubridora de las diferencias de clase. Extraída y perfilada - en buena medida- de su ejercicio como gerente deportivo. Extrapola la gestión desde el deporte a la política (ALTUVE-MEJÍA, 2018).

En su estrategia electoral redimensionó - en una escala nunca antes conocida - la presencia del candidato intercambiando con los electores como ayuda para crear mejores políticas públicas, logrando encarnar en su figura la novedad en el estilo de gobierno, asociado al dialogo, humildad, proximidad, transparencia. Publicó contenidos cumpliendo "el rol de padre y esposo", presentándose como un hombre común, igual a cualquier otro, "creándose la imagen de un político con sensibilidad y accesible" (ALTUVE-MEJÍA, 2018, p.504-505). Combinándola magistralmente con la dotación de una épica y heroísmo único que lo diferenciaba de los demás candidatos y lo hacía acreedor de las condiciones para ser presidente. En ese sentido:

- Contó en primera persona - con el título de El gran desafío - la experiencia de su secuestro ocurrido el 24-08-1991, donde su protagonismo está asociado a la labor en el Boca Juniors, publicándola en julio 2015 con rotundo éxito mediático. Su heroísmo se 
refuerza actuando como protagonista en Boca Juniors 3D La Película (documental armado en las islas de edición del canal Fox Sports), que el mismo Macri financió y difundió (ALTUVE-MEJÍA, 2018).

- Fue el candidato que más y mejor incorporó la comunicación digital, articulándola exitosamente con los medios convencionales. Facebook concluyó que "Argentina entró en el top 5 de las elecciones más comentadas con 90 millones de interacciones (likes, publicaciones, comentarios y contenido compartido", debido a Macri (ARIZA, 2016, p. 6).

- Sus intervenciones en la prensa gráfica y audiovisuales eran publicados en los medios digitales, popularizó la participación ciudadana mediante hashtags, propuso a los usuarios que expusieran ideas y opiniones: "compartir fotos con la leyenda \#YoVotoaMM y comentar las razones porque lo hacían MacrienAdosVoces, \#MacriConFantino, entre otros, eran publicaciones que inundaron su red social". Esta confluencia de medios le permitió compartir los comentarios más significativos en las redes sociales, que fueron utilizadas como una plataforma más para informar y continuar con la propaganda electoral aun estando prohibida, aprovechando los vacíos de regulación legal en los medios digitales (ARIZA, 2016).

- Los adversarios erraron al reducir a un simple dato curricular la gestión de Macri en el Boca Juniors y no enfrentar su incorporación del deporte a la campaña electoral. Existían elementos para enfrentarla con algunas posibilidades de éxito o que disminuyera el efecto positivo que tenía sobre la población, teniendo en cuenta que los hechos de corrupción y actos delictivos en los que incurrió en el Boca Juniors eran conocidos y de manejo público. Más allá de su incompetencia, no lo hicieron porque también estaban convencidos de la actuación exitosa de Macri en el Boca Juniors. Y por lo tanto no podían atacar lo que ellos también consideraban y concebían como positivo (ALTUVE-MEJÍA, 2017).

Importante registrar también que Macri logró el respaldo a su exitosa campaña electoral de Tévez, Riquelme, Ramón Díaz, Francescoli, Basile, Del Potro, entre otras figuras deportivas mundiales.

\section{CONCLUSIONES}

El deporte es una EDTEE que funciona con la lógica, dinámica y orientación de cualquier transnacional con algunas especificidades y cuyo objetivo es producir un espectáculo cotidiano, masivo y rentable.

El poder en la EDTEE se concentra en: a) La ODM por su condición de gobierno del deporte mundial y por organizar-gestionar una parte fundamental del espectáculo encabezado por los JO y el Mundial de Fútbol. b) Un reducido número de Estados nacionales encabezado por el Grupo de los Nueve:

- Cuyas transnacionales organizadoras-gestoras, de artículos e implementos deportivos medios de comunicación y patrocinantes, se apropian de los más elevados volúmenes de ingreso producidos por el espectáculo.

- Cuyos deportistas-competidores son los ganadores de la mayoría de los eventos más importantes.

Lula y Dilma Rouseff previeron realizar el Mundial 2014 y los JO 2016 financiado con elevada inversión de capital privado, estableciendo determinados privilegios que garantizaban elevados ingresos para la FIFA, COI y sus Socios transnacionales patrocinantes. El problema fue que la situación económica desmejoró con la disminución de los ingresos estatales, la inversión privada no se produjo, 
aumentó considerablemente el costo de los eventos por la corrupción políticoempresarial y se mantuvieron inalterables las prebendas e ingreso de los principales beneficiarios. El Estado disponiendo de menos ingresos y prácticamente sólo tuvo que asumir un financiamiento mucho más costoso, puede decirse que el Mundial y los JO descapitalizaron las arcas públicas de Brasil.

Fueron notorios los privilegios fiscales, comerciales y amplias y diversas competencias, que el Estado le otorgó a los organizadores-gestores del Mundial y JO, FIFA y COI respectivamente, y a sus Socios transnacionales patrocinantes. Siendo todavía mucho más difundidas y evidentes las promesas del gobierno, la FIFA y el COI, de que producirían amplios beneficios, progreso, orgullo, ratificarían la condición de potencia del país y tendrían un fuerte impacto positivo para el desarrollo económico por la inversión privada que se realizaría, creando un amplio apoyo popular a ambos eventos.

Lo prometido no se cumplió, la esperanza e ilusión de progreso y ventajas económicas progresivamente se fueron desvaneciendo. El costo del Mundial y JO duplicó lo estimado inicialmente por los desmedidos privilegios de la FIFA, el COI y sus Socios transnacionales patrocinantes y por la corrupción político-empresarial en la construcción y remodelación de la infraestructura. Al mismo tiempo que la inversión privada no se produjo.

Lejos de obtenerse los beneficios prometidos, se sufrió la violenta política de desalojos y reconversiones urbanísticas aplicada para disponer de espacios para los dos eventos, y se agravó considerablemente el problema de la vivienda.

Las denuncias de los elevados costos que tenía para las arcas públicas el Mundial y cuestionamientos a su realización, quejas contra la FIFA, fueron incorporadas como elementos movilizadores que coadyuvaron a las masivas y multitudinarias protestas en 2013, se articularon con exigencias de solución de necesidades básicas, demandas de reivindicaciones muy específicas para mejorar la calidad de vida. Contribuyeron de alguna manera a que la aprobación del gobierno cayera a la mitad y que la reelección de Dilma Rouseff, en 2014, fuese muy estrecha.

El rechazo al Mundial contribuyó con el cuestionamiento (o puesta en duda) del carácter popular-democrático del final del primero y del segundo gobierno de Dilma Rouseff, al evidenciar que favorecía y mantenía inalterables los beneficios de los poderosos, mientras engañaba, decepcionaba a su pueblo porque no cumplió lo prometido y agravó sus problemas al sufrir la violenta política de desalojos y reconversiones y agudizarse el problema de la vivienda. Contribuyó con el debilitamiento político de Dilma e incidió favorablemente en su destitución en 2016, episodio que es el punto de quiebre de la democracia brasileña y marca el retorno del neoliberalismo a Brasil con Michel Temer en el poder.

En la victoriosa campaña electoral que llevó al poder a Bolsonaro significando la profundización del proyecto neoliberal en Brasil, el deporte fue uno de los escenarios donde mejor operó la auténtica revolución tecno-política, al lograr registrar, difundir y propagar masivamente a través de las redes sociales la presencia y protagonismo del candidato en los estadios y el apoyo de famosos deportistas-competidores.

Para Macri, ejercer del 1995 al 2007 la presidencia-gerencia del Club de Fútbol Boca Juniors de manera exitosa, fue el trampolín para su ingreso a la política, ha signado su carrera y lo incorporó a la victoriosa campaña electoral presidencial 2015 que significó el retorno del neoliberalismo en Argentina.

El centro de la propuesta política de Macri fue la gestión, extraída y perfilada - en buena medida - a raíz de su ejercicio como gerente deportivo. Extrapola la "gestión" desde el deporte a la política. 
Eloy Altuve-Mejía

Combinó magistralmente la imagen de un político con sensibilidad, accesible, cercano, igual a cualquier ciudadano, con la dotación de una épica y heroísmo único asociada- alimentada fuertemente por su labor en el Boca Juniors y que lo diferenciaba de los demás candidatos y convertía en el acreedor de las condiciones para ser presidente de la nación. Y utilizó acertadamente los medios para comunicar exitosamente su propuesta basada en la gestión y la articulación perfecta de igualdad con diferencia.

Los adversarios erraron al no enfrentar la incorporación del deporte a la campaña electoral macrista. Más allá de su incompetencia, no lo hicieron porque también estaban convencidos de la actuación exitosa de Macri en el Boca Juniors. Y por lo tanto no podían atacar lo que ellos también consideraban y concebían como positivo. 


\section{REFERENCIAS}

ALTUVE-MEJÍA, E. Papel del deporte en la irrupción fascista en Brasil 2018: ¿Cómo el deporte fue convertido en elemento importante de la campaña de Bolsonaro? ¿De qué forma el Mundial de Fútbol y Juegos Olímpicos contribuyeron con la destitución de Dilma Rouseff, el punto de quiebre de la democracia? ATHLOS, v. 16, n. 8, p. 82-142, 2019.

ALTUVE-MEJÍA, E. Globalización, neoliberalismo, política pública y poder. Papel del deporte en el retorno del proyecto neoliberal. Centro Experimental de Estudios Latinoamericanos "Dr. Gastón Parra Luzardo", Universidad del Zulia, Maracaibo-Venezuela. Edición Digital. Quito: CIED, 2018.

ALTUVE-MEJÍA, E. Papel del deporte en el regreso del neoliberalismo como proyecto político y económico en la segunda década del siglo XXI: Brasil y Argentina. Revista da ALESDE, v. 7, n. 1., p. 51-78, 2017.

ALTUVE-MEJÍA, E. Deporte, globalización y política. Medellín-Colombia: Editorial Kinésis, 2016.

ARIZA, A. Mauricio Macri: claves de una campaña virtual exitosa. En: CONGRESO NACIONAL/CONGRESO INTERNACIONAL SOBRE DEMOCRACIA， 12./5., 2016, Actas... Rosario, 2016.

CARCIONE, C. El fenómeno Bolsonaro. ¿Punto de llegada o momento de aceleración de la crisis? Caracas. Disponible en: <https://www.aporrea.org/internacionales/a271635.html >. Accedido en: 11 nov. 2018.

GENTILI, P. Golpe en Brasil. Consejo Latinoamericano de Ciencias Sociales (CLACSO). Buenos Aires: Octubre Editorial. 2016.

LIGA DE FÚTBOL PROFESIONAL DE ESPAÑA. Patrocinadores. Madrid. Disponible en: < https://www.laliga.es/patrocinadores >. Accedido en: 01 feb. 2019.

PREMIER LEAGUE. Socios $\mathbf{y}$ licenciatarios oficiales. Londres. Disponible en: < https://www.premierleague.com/partners >. Accedido en: 01 feb. 2019.

ZIMBALIST, A. El negocio económico detrás de la organización de los JO y el Mundial de Fútbol. Madrid: Ediciones Akal, 2016.

Recibido en: 02 feb. 2019.

Aprobado en: 17 abr. 2019. 